\title{
Natural Deep Eutectic Solvent Extraction of Flavonoids of Scutellaria baicalensis as a Replacement for Conventional Organic Solvents
}

\author{
Wim Wouter Oomen ${ }^{1,+}$, Paloma Begines ${ }^{1,+}$, Natali Rianika Mustafa ${ }^{1}$, Erica G. Wilson ${ }^{1}$, \\ Robert Verpoorte $^{1}$ and Young Hae Choi ${ }^{1,2, *(D)}$ \\ 1 Natural Products Laboratory, Institute of Biology, Leiden University, Sylviusweg 72, 2333 BE Leiden, \\ The Netherlands; wim.oomen@hotmail.com (W.W.O.); pbegines@us.es (P.B.); \\ mustafa@chem.leidenuniv.nl (N.R.M.); erica.g.wilson@gmail.com (E.G.W.); \\ verpoort@chem.leidenuniv.nl (R.V.) \\ 2 College of Pharmacy, Kyung Hee University, Seoul 02447, Korea \\ * Correspondence: y.choi@chem.leidenuniv.nl; Tel.: +31-71-527-4510 \\ + These authors equally contributed to the article.
}

Academic Editor: Dimitris P. Makris

Received: 6 January 2020; Accepted: 30 January 2020; Published: 31 January 2020

\begin{abstract}
Natural deep eutectic solvents (NADES) are a type of ionic liquid (IL) or deep eutectic solvent (DES), the ingredients of which are exclusively natural products (non-toxic and environmentally friendly). Here, we explore the potential of NADES as an alternative to conventional organic solvents (e.g., aqueous methanol or ethanol) for the extraction of flavonoids from Scutellaria baicalensis stem bark to investigate their extractability depending on structural variation. Four NADES, each containing citric acid in combination with $\beta$-alanine, glucose, xylitol, or proline (at a molar ratio of 1:1), and a variable amount of water, were used to extract the flavonoid aglycones: baicalein (1), scutellarein (3), wogonin (5), and oroxylin A (7), and their glycosides, baicalin (2), scutellarin (4), wogonoside (6) and oroxyloside (8) from the powdered bark of S. baicalensis. The chemical profile and yield of the extracts were determined using HPTLC and HPLC. The extractability of individual flavonoids was found to be influenced by the concentration of water $(20-60 \%, w / w)$ in the NADES. Among the tested flavonoids, the extraction yield of baicalein (1), scutellarein (3), wogonin (5), oroxylin A (7) with NADES was 2 to 6 times that of aqueous methanol. However, the amount of their corresponding glycosides (baicalin (2), wogonoside (6) and oroxyloside (8)) extracted with NADES was only 1.5-1.8 times higher than with aqueous methanol. Interestingly, the more hydrophilic glycosides were less extracted than their corresponding aglycones despite the high hydrophilicity of the NADES. These results prove that NADES may be used for extraction of compounds with a wide range of hydrophilicity.
\end{abstract}

Keywords: NADES; extraction; ionic liquid; lipophilic compounds; HPTLC; HPLC; phenolic compounds

\section{Introduction}

Organic solvents are considered to be irreplaceable in many industries, being used in many processes e.g., for the production of medicines, extraction of colorants and dyes, purification of compounds from diverse matrixes and numerous other applications. Unfortunately, despite their toxicity and the environmental hazard they pose, organic solvents are still used in great amounts, with high costs involved in their waste disposal. It has thus been some time now that the industry has been attempting to replace them with greener technology. There are four major strategies to avoid the use of conventional harmful or toxic organic solvents. These are: 1) the use of heterogeneous catalysts, 2) water, 3) supercritical fluids or 4) ionic liquids (ILs) [1], as well as 5) a recently emerging 
field, the application of nanoparticles to the extraction of various samples, which has been reviewed for environmental applications [2], food toxins [3] and biological fluids [4]. With the exception of the use of catalysts, all the alternatives are associated with the use of non- or at least less toxic solvents. However, these main strategies have not had the expected success and the search for a really 'green' substitute for the currently used solvents is still needed. Thus, over the last decade, in the field of green technology, ILs and their related technologies have been actively applied across a wide range of fields [5].

Ionic liquids are ionic mixtures, the state of which changes from solid to liquid around or below $100{ }^{\circ} \mathrm{C}$ [1]. One of the main characteristics of ILs is that they have no detectable vapor pressure, and consequently do not evaporate in ambient conditions. ILs are characterized by the existence of ionic bonds between their components, which require at least one cation and one anion. However, the application of ILs in the food industry is limited because many of them are based on imidazolium and pyridinium species and are therefore toxic. A subclass of solvents that have similar eutectic properties are deep eutectic solvents (DES), first described in 2001 by Abbot and his colleagues [6]. A DES is a liquid that is formed when at least two certain solid compounds are mixed in conditions that lower their melting points to a point low enough to form a eutectic mixture [7,8]. In 2011, Choi and colleagues proposed that there might be DES-like media in nature playing many biological roles and hypothesized that this new kind of DES, they named natural deep eutectic solvents (NADES) might be present in living organisms. Natural deep eutectic solvents are natural solvents based on the principle of ILs and DES, but primarily composed of natural compounds. They thus constitute a very promising option for green chemistry and are candidates to replace the toxic organic solvents [9].

Among many NADES applications, the most extensively studied refer to the extraction of natural products. In particular, very diverse natural phenolic compounds have been extracted with NADES, very successfully in most cases. For example, extraction of anthocyanins from grape skins with a NADES consisting of citric acid-maltose (4:1; mole/mole) showed a yield that practically doubled that obtained with $80 \%$ methanol [10]. Other successful applications include the extraction of trans-resveratrol from roots of Polygoni cuspidati [11], C-glucosyl quinochalcones from flowers of Carthamus tinctorius [12], anthocyanins from grape pomace [13] and phenolic antioxidants from Greek medicinal plants [14]. Alongside the extraction yields, Dai and colleagues also found that NADES could not only increase the extraction yield but also the stability target compounds [15]. Moreover, by tailoring the water concentrations in NADES, Dai and colleagues laid the basis for a manageable way of applying NADES in food processing, enzymatic reactions and pharmaceutical and cosmetic applications [16]. Recent applications of NADES to diverse fields have been reviewed by Vanda and colleagues [17].

In this study, the feasibility of using NADES to extract phenolics, particularly flavonoids, was further investigated. Roots of Scutellaria baicalensis (Labiatae) were selected as model material. This plant is one of the most popular herbal drugs in traditional Chinese medicine (TCM) and is often used for the treatment of cardiovascular disease and bleeding disorders as well as for some infectious diseases $[18,19]$. The main active compounds of the plant have been reported to be flavonoids and so far, over 40 analogues have been identified in this plant material [20].

Although NADES are very promising solvents, some of their inherent features such as high viscosity and low vapor pressure make their evaluation difficult. Thus, a sample preparation procedure and analytical method that can circumvent these difficulties is needed to analyze the extracts. Recently, a method based on high performance thin layer chromatography (HPTLC) coupled to multivariate data analysis (MVDA) was developed for the analysis of NADES plant extracts [21]. Previously, for the analysis of plant extracts, HPTLC-based methods were shown to be easily applied to both targeted and non-targeted studies aimed at obtaining their chemical profiles [22,23]. For NADES extracts, the HPTLC-based method provided a quick and reliable method to perform the 'fingerprint' analyses of the extracts, as well as a broad coverage of metabolites [21]. 
In this study, roots of $S$. baicalensis were extracted with several NADES, followed by chemical assessment by HPTLC in an untargeted manner. In addition to HPTLC analysis, a target analysis of the major flavonoids was performed by HPLC-UV.

The results of this study allowed us to evaluate both the extraction efficiency of the selected NADES and their extraction profiles. Apart from the extraction capacity of the different NADES, the effect of water addition in NADES was also evaluated.

\section{Results and Discussion}

In this study, four different NADES and their capability to extract flavonoids from S. baicalenisis was tested. Following previous work by González and colleagues [24], all four selected NADES were based on citric acid because they were non-toxic and had potential to extract phenolics. The selected NADES were 1:1 molar ratio mixtures of $\beta$-alanine-citric acid (N1), glucose-citric acid (N2), xylitol-citric acid (N3) and proline-citric acid (N4). All of the components were apt for consumption, to prove that NADES-based extracts can be used directly for food or pharmaceutical applications. Among the many flavonoids present in Scutellaria baicalensis, eight of the major components were chosen: baicalein (1), scutellarein (3), wogonin (5), oroxylin A (7) and their glycosides, baicalin (2), scutellarin (4), wogonoside (6) and oroxyloside (8) [25] (Figure 1).

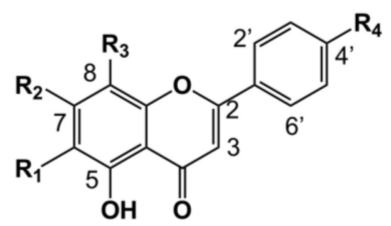

$$
\begin{aligned}
& \mathrm{R}_{1}=\mathrm{OH}, \mathrm{R}_{2}=\mathrm{OH}, \mathrm{R}_{3}=\mathrm{H}, \mathrm{R}_{4}=\mathrm{H} \text { baicalein }(\mathbf{1}) \\
& \mathrm{R}_{1}=\mathrm{OH}, \mathrm{R}_{2}=\text { glucuronide, } \mathrm{R}_{3}=\mathrm{H}, \mathrm{R}_{4}=\mathrm{H} \text { baicalin }(\mathbf{2}) \\
& \mathrm{R}_{1}=\mathrm{OH}, \mathrm{R}_{2}=\mathrm{OH}, \mathrm{R}_{3}=\mathrm{H}, \mathrm{R}_{4}=\mathrm{OH} \text { scutellarein }(\mathbf{3}) \\
& \mathrm{R}_{1}=\mathrm{OH}, \mathrm{R}_{2}=\text { glucuronide, } \mathrm{R}_{3}=\mathrm{H}, \mathrm{R}_{4}=\mathrm{OH} \text { scutellarin }(\mathbf{4}) \\
& \mathrm{R}_{1}=\mathrm{H}, \mathrm{R}_{2}=\mathrm{OH}, \mathrm{R}_{3}=\mathrm{OCH}_{3}, \mathrm{R}_{4}=\mathrm{H} \text { wogonin }(\mathbf{5}) \\
& \mathrm{R}_{1}=\mathrm{H}, \mathrm{R}_{2}=\mathrm{OH}, \mathrm{R}_{3}=\mathrm{OCH}_{3}, \mathrm{R}_{4}=\mathrm{H} \text { wogonoside }(\mathbf{6}) \\
& \mathrm{R}_{1}=\mathrm{OCH}_{3}, \mathrm{R}_{2}=\mathrm{OH}, \mathrm{R}_{3}=\mathrm{H}, \mathrm{R}_{4}=\mathrm{H} \text { oroxylin } \mathrm{A}(\mathbf{7}) \\
& \mathrm{R}_{1}=\mathrm{OCH}_{3}, \mathrm{R}_{2}=\text { glucuronide, } \mathrm{R}_{3}=\mathrm{H}, \mathrm{R}_{4}=\mathrm{H} \text { oroxyloside }(\mathbf{8})
\end{aligned}
$$

Figure 1. Chemical structures of the flavonoids of Scutellaria baicalensis employed in the study.

The inherent viscosity of most NADES requires a relatively long extraction time and can lead to some difficulties in post-extraction procedures, e.g., residual solvents in the extracts interfering with the analysis. There are two ways to solve this problem: to increase the working temperature or add a certain percentage of water. It has been observed that even adding very small amounts of water can result in a dramatic decrease in the viscosity of most NADES. Thus, $10 \%$ of water was added to the NADES as suggested by Dai and colleagues [16]. This proved to successfully lower the viscosity and partly solve the problems during extraction, but the recovery of metabolites from the NADES extract using a solid phase extraction (SPE) column still proved to be rather difficult even with this relatively lower viscosity.

The comparison of extraction patterns was firstly performed using HPTLC whereas HPLC was applied for quantitative analysis by targeting the selected flavonoids. The advantage of using HPTLC is that it allows the simultaneous analysis of multiple samples on one plate, facilitating their comparison. A disadvantage is that given the difference in hydrophilicity of the targeted flavonoid aglycones and their glycosides, it is no less hard to obtain an acceptable resolution on the same plate with the same chromatographic system. Thus, it is not possible to capture all flavonoids in one HPTLC image. To solve the problem, we ran the HPTLC experiment with two different mobile phases, managing to identify extraction 'fingerprints' which were then categorized based on principle component analysis (PCA). PCA is necessary to analyze multiple variables in the HPTLC image (e.g., Rf-value, color and intensity) in order to reveal the difference among the observed groups (different extraction profiles). It was not 
possible to separate all flavones and their glycosides with the HPTLC system, so that quantitative analysis of the content of each of these compounds was done using HPLC. The HPLC results confirmed the presence of seven of the targeted flavonoids (baicalein (1), scutellarein (3), wogonin (5), oroxylin A (7) and the glycosides baicalin (2), wogonoside (6) and oroxyloside (8)), while the glycoside scutellarin (4) was undetectable in all of the extracts. This differed from the results obtained by Liu and colleagues, who reported the presence of scutellarin in Scutellaria baicalensis [25].

\subsection{Comparison of Extraction Profiles and Yields between Different NADES and Organic Solvents}

In this work, four selected edible NADES were tested for their flavonoid extraction capability. Following the determination of the overall extraction yield of the targeted flavonoids, the two most efficient NADES were selected for further studies. The organic solvents used as controls were $80 \%(v / v)$ aqueous methanol and $70 \%(v / v)$ aqueous ethanol. Firstly, the HPTLC fingerprint extraction pattern was developed as shown in Figure 2. At first sight, the control aqueous ethanol and methanol profiles were more intense in comparison to the NADES profiles. However, a closer inspection of the profiles, revealed differences in the extraction profiles of the NADES and the control extracts with an increase in certain flavonoids. The HPTLC bands were further analyzed by PCA after data processing and the score plot revealed a similarity in the extraction capacity of the two control solvents and a similarity amongst the different NADES (Figure 3a). Interestingly, the NADES, N4 with citric acid-proline (1:1, mole/mole), was found to have a slightly different profile to those of the other NADES, N1 (citric acid- $\beta$-alanine, 1:1, mole/mole), N2 (citric acid-glucose, 1:1, mole/mole), and N3 (citric acid-xylitol, 1:1, mole/mole). Further observations on the similarities and differences between the extracts, the HPLC data targeted for the selected flavonoids was analyzed by PCA, for which the peak intensities of eight flavonoids were used as variables (Figure 3b). The score plot of PCA showed that N1 and N4 had clearly distinct extraction profiles.

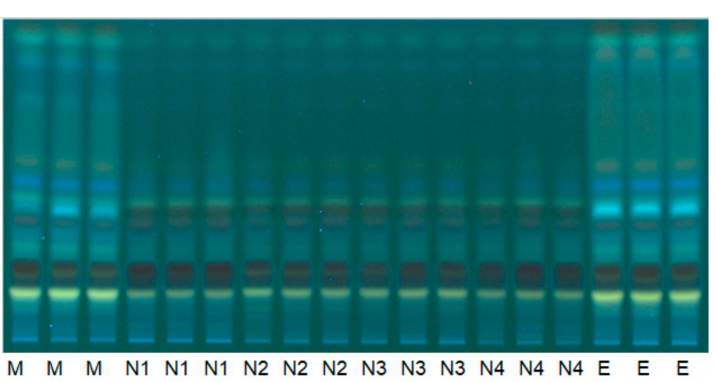

(a)

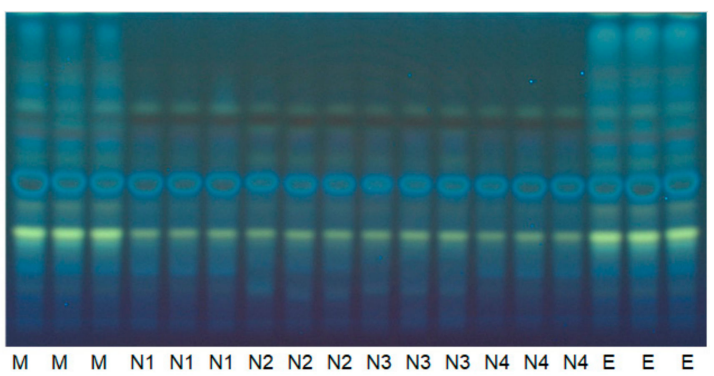

(b)

Figure 2. High-performance thin-layer chromatograms (HPTLC) of Scutellaria baicalensis bark extracted with $80 \%$ methanol in water, $70 \%$ ethanol in water and natural deep eutectic solvents (NADES) visualized at $366 \mathrm{~nm}$ after derivatization with natural products reagent (NPR) with two different mobile phase conditions. (a) Chromatogram obtained with the mobile phase: ethyl acetate, methanol, formic acid, water (EMFW, 20:2.7:0.5:2, ratio $v / v / v / v)$; (b) Chromatogram obtained with the mobile phase: ethyl acetate, formic acid, acetic acid and water (EFAW, 100:11:11:27, v/v/v/v). M: 80\% methanol in water extract $(v / v), E$ : $70 \%$ ethanol in water $(v / v)$, N1-N4: NADES with the addition of $10 \%$ water $(w / w)$. N1: citric acid- $\beta$-alanine (1:1, mole/mole). N2: citric acid-glucose (1:1, mole/mole). N3: citric acid-xylitol (1:1, mole/mole). N4: citric acid-proline (1:1, mole/mole). 


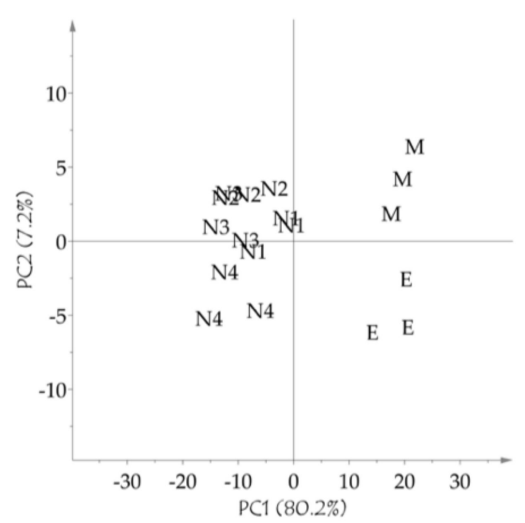

(a)

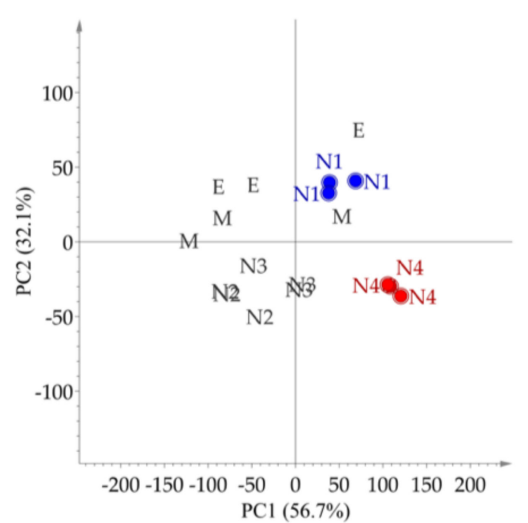

(b)

Figure 3. Score plot of principle component analysis of the HPTLC (a) and HPLC (b) data obtained from the extracts of $80 \%$ aqueous methanol, $70 \%$ aqueous ethanol, and natural deep eutectic solvent (NADES) extracts of Scutellaria baicalensis bark. M: 80\% methanol in water extract ( $v / v)$, E: 70\% ethanol in water $(v / v), \mathrm{N} 1-\mathrm{N} 4$ : NADES with the addition of $10 \%$ water $(w / w)$. N1: citric acid- $\beta$-alanine (1:1, mole/mole). N2: citric acid-glucose (1:1, mole/mole). N3: citric acid-xylitol (1:1, mole/mole). N4: citric acid-proline (1:1, mole/mole).

The HPLC analyses were used to compare the extraction profile of each NADES and their quantitative composition in targeted flavonoids. The yield of total flavonoids obtained with each NADES (N1 to N4), based on the peak integrals of flavonoids, were $80 \%-170 \%$ that of aqueous methanol or ethanol extracts. A higher yield of the targeted flavonoids was also observed in N1 and N4 extracts so these NADES were selected for further studies involving the effect of water addition.

\subsection{Effect of Different Water Concentrations in NADES on Flavonoid Extraction}

It has been reported that water content is one of the factors that most affects NADES extraction capacity [16]. Thus, in this second part, the influence of different water concentrations in N1 and N4, which showed promising results, on their flavonoid extraction capacity was explored. The correlations between solubility or extractability, and water content are not necessarily linear and, in many cases, the optimum solubility or extractability is found only at a specific water content in NADES [16].

This test was carried out by extracting the sample with the two best performing NADES, N1 and $\mathrm{N} 4$, to which increasing amounts of water $(20 \%-60 \%, w / w)$ were added. The HPTLC fingerprints clearly revealed the extent of the influence of the water content on the yield of the individual compounds (Figure 4). A possible explanation for this is the increased hydrophilicity provided by the water content in the NADES that favors the extractability of the flavonoids. To investigate the effect of water on the extraction profile, orthogonal partial least square (OPLS) modeling was applied to the data of HPTLC and HPLC, for which the percentage of added water or the kind of NADES (N1 and N4) was used as the Y-variable and the combined HPLC and HPTLC data were treated as single X-data. As shown in Figure 5A,B, the extraction profile was greatly influenced by the NADES character (N1 and N4) and water content. The two OPLS models, each of which is for water effect and the characters of NADES, respectively were combined and the SUS plot was employed to interpret the individual effect on each flavonoid (Figure 5C, X-axis: NADES characters, $Y$-axis: water content). Most glycosides were influenced more by water content than aglycones, except for scutellarein (3). Interestingly, each NADES showed different effects; N1 was found to extract more glycosides such as baicalin (2), wogonoside (6) and oroxyloside (8), but N4 showed better extraction yields for aglycones (3), wogonin (5) and oroxylin A (7). 

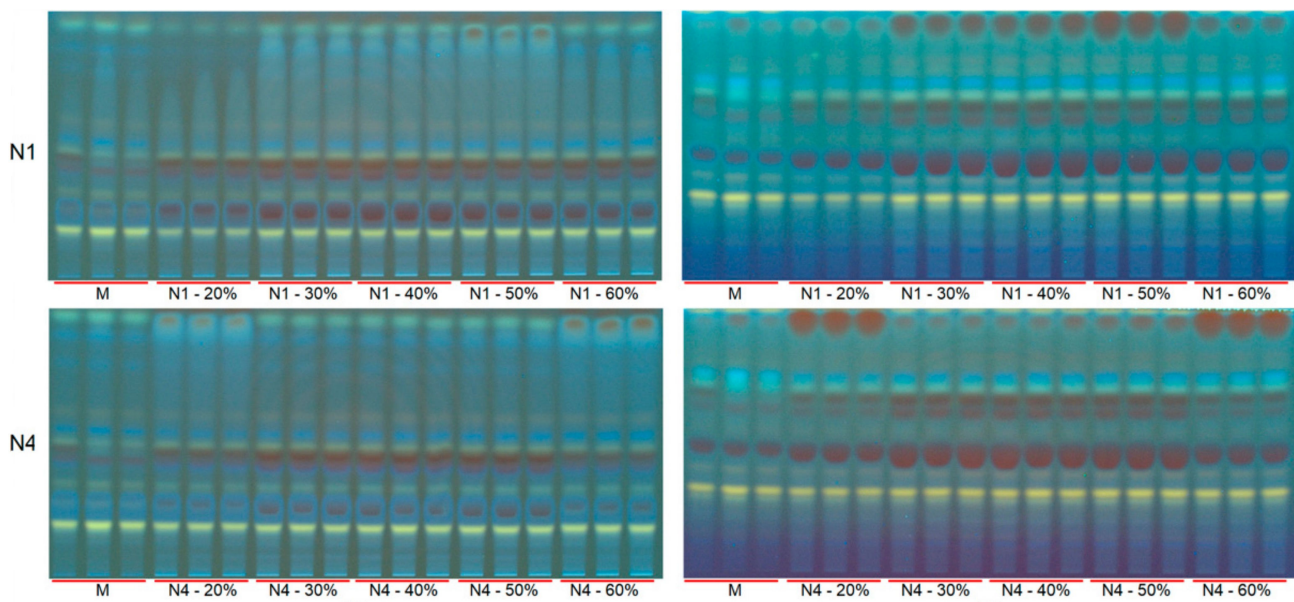

(a)

(b)

Figure 4. High-performance thin-layer chromatograms (HPTLC) of extracts of S. baicalensis bark obtained with $80 \%$ methanol in water and two NADES with different concentrations of water visualized at $366 \mathrm{~nm}$ after derivatization with natural products reagent (NPR) with two different mobile phase conditions. (a) Chromatogram obtained with mobile phase: ethyl acetate, methanol, formic acid, water (EMFW, 20:2.7:0.5:2, ratio $v / v / v / v)$; (b) Chromatogram obtained with mobile phase: ethyl acetate, formic acid, acetic acid and water (EFAW, 100:11:11:27, v/v/v/v). M: 80\% aqueous methanol (v/v), N1-20: citric acid- $\beta$-alanine (1:1, mole/mole) with the addition of $20 \%(w / w), N 1-30$ : citric acid- $\beta$-alanine (1:1, mole/mole) with the addition of $30 \%(w / w)$, N1-40: citric acid- $\beta$-alanine (1:1, mole/mole) with the addition of $40 \%(w / w), \mathrm{N} 1-50$ : citric acid- $\beta$-alanine $(1: 1, \mathrm{~mole} / \mathrm{mole})$ with the addition of $50 \%(w / w)$, N1-60: citric acid- $\beta$-alanine (1:1, mole/mole) with the addition of $60 \%(w / w)$, N4-20: proline-citric acid (1:1, mole/mole) with the addition of $20 \%(w / w)$, N4-30: proline-citric acid (1:1, mole/mole) with the addition of $30 \%(w / w), N 4-40$ : proline-citric acid (1:1, mole/mole) with the addition of $40 \%(w / w)$, N4-50: proline-citric acid (1:1, mole/mole) with the addition of 50\% (w/w), N4-60: proline-citric acid $(1: 1$, mole/mole) with the addition of $60 \%(w / w)$.

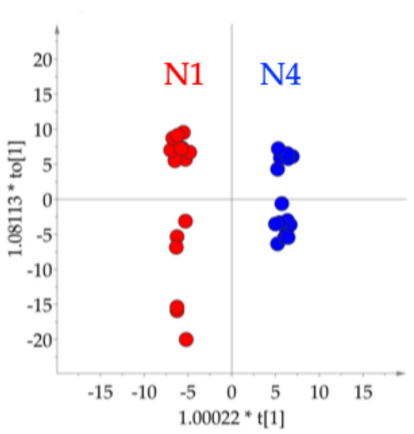

(a)

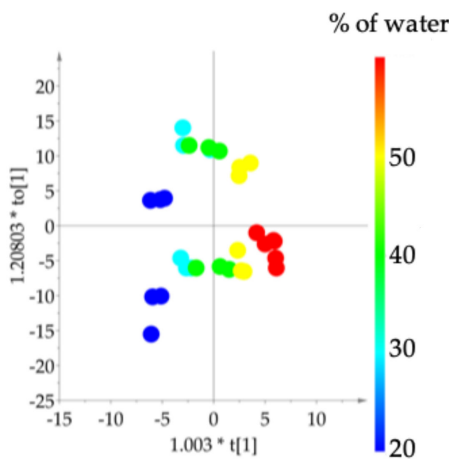

(b)

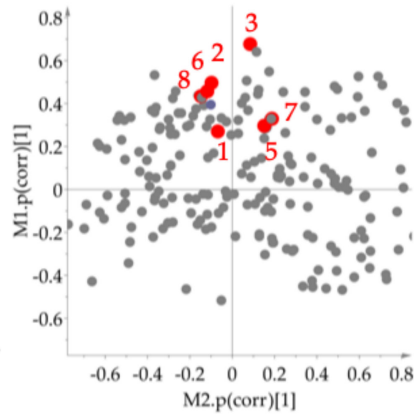

(c)

Figure 5. Orthogonal partial least square modeling of HPTLC and HPLC data of Scutellaria baicalensis roots with the kind of NADES (N1 and N4) and the percentage of water added to NADES. (a) Score plot of OPLS-DA for the effect of the kind of NADES (N1 and N4), (b) Score plot of OPLS for the effect of the percentage of added water, (c) SUS plot to combine two models (the kinds of NADES and the percentage of water). N1: citric acid- $\beta$-alanine (1:1, mole/mole). N4: citric acid-proline (1:1, mole/mole). Compounds in SUS plot (c): baicalein (1), scutellarein (3), wogonin (5), oroxylin A (7) and their glycosides, baicalin (2), wogonoside (6) and oroxyloside (8).

The HPLC analyses of the extracts showed that, as expected, the flavonoid aglycones and their corresponding glycosides were not best extracted at the same water concentration. Unexpectedly, the higher percentage of water did not increase the yield of the more hydrophilic glycosides. There might be several reasons for this phenomenon. The addition of water does not greatly change hydrophilicity 
of NADES because the components of NADES are already hydrophilic enough and thus not affected by the addition of water. Secondly, the extraction or solubilization of flavonoids does not follow the increase of hydrophilicity of the solvents in a proportional manner, that is, an optimum hydrophilicity might be attained with a specific amount of water Actually, some phenolics are best extracted with specific water contents [16].

All of the tested NADES were able to extract flavonoids, some being more efficient than conventional organic extraction (Table 1). The maximum increase in the yield of baicalein (1), scutellarein (3), wogonin (5) and oroxylin A (7) was approximately 3-5 times that of 80\% methanol extraction. On the other hand, the corresponding glycosides of these flavonoids showed a lower increase in their extraction yields with NADES of approximately 1.5-fold when compared with the organic solvent extraction.

Table 1. Yield of individual flavonoids extracted from Scutellaria baicalensis root in NADES solvents N1 (citric acid- $\beta$-alanine, 1:1) and N4 (citric acid-proline. 1:1) with different water concentrations. Solvents in $\mu \mathrm{g} / \mathrm{mg}$ plant extract \pm SD based on triplicates.

\begin{tabular}{cccccccc}
\hline Solvent & $\begin{array}{c}\text { baicalein } \\
\text { (1) }\end{array}$ & $\begin{array}{c}\text { baicalin } \\
\mathbf{( 2 )}\end{array}$ & $\begin{array}{c}\text { scutellarein } \\
\mathbf{( 3 )}\end{array}$ & $\begin{array}{c}\text { wogonin } \\
\mathbf{( 5 )}\end{array}$ & $\begin{array}{c}\text { wogonoside } \\
\mathbf{( 6 )}\end{array}$ & $\begin{array}{c}\text { oroxylin A } \\
\text { (7) }\end{array}$ & $\begin{array}{c}\text { oroxyloside } \\
\text { (8) }\end{array}$ \\
\hline $\mathrm{M}$ & $1.4 \pm 0.2$ & $27.8 \pm 1.8$ & $6.4 \pm 0.7$ & $14.1 \pm 1.2$ & $14.9 \pm 0.4$ & $1.1 \pm 0.2$ & $2.0 \pm 0.3$ \\
$\mathrm{E}$ & $1.3 \pm 0.4$ & $24.9 \pm 4.8$ & $5.1 \pm 1.5$ & $11.3 \pm 4.0$ & $21.9 \pm 7.8$ & $1.3 \pm 0.5$ & $2.4 \pm 0.8$ \\
$\mathrm{~N} 1-20 \%$ & $1.4 \pm 0.4$ & $26.2 \pm 4.8$ & $3.8 \pm 0.2$ & $8.8 \pm 0.7$ & $24.1 \pm 2.2$ & $1.0 \pm 0.3$ & $2.0 \pm 0.3$ \\
$\mathrm{~N} 1-30 \%$ & $1.8 \pm 0.3$ & $25.3 \pm 2.1$ & $6.4 \pm 0.4$ & $17.1 \pm 0.8$ & $51.9 \pm 0.4$ & $2.4 \pm 0.2$ & $4.8 \pm 0.3$ \\
$\mathrm{~N} 1-40 \%$ & $2.7 \pm 0.8$ & $39.4 \pm 9.5$ & $7.5 \pm 0.9$ & $18.6 \pm 1.3$ & $59.4 \pm 4.5$ & $2.9 \pm 0.4$ & $5.4 \pm 0.7$ \\
$\mathrm{~N} 1-50 \%$ & $3.0 \pm 0.6$ & $38.1 \pm 4.6$ & $6.8 \pm 0.4$ & $16.7 \pm 0.4$ & $73.3 \pm 0.9$ & $4.7 \pm 1.0$ & $8.6 \pm 0.5$ \\
$\mathrm{~N} 1-60 \%$ & $2.6 \pm 0.3$ & $37.9 \pm 1.8$ & $6.9 \pm 0.3$ & $17.1 \pm 0.8$ & $39.6 \pm 1.5$ & $2.2 \pm 0.3$ & $3.9 \pm 0.3$ \\
$\mathrm{~N} 4-20 \%$ & $2.3 \pm 0.3$ & $28.8 \pm 1.9$ & $3.8 \pm 0.4$ & $8.2 \pm 0.7$ & $73.9 \pm 1.7$ & $6.2 \pm 0.5$ & $9.3 \pm 0.6$ \\
$\mathrm{~N} 4-30 \%$ & $2.0 \pm 0.5$ & $31.8 \pm 5.5$ & $6.6 \pm 0.5$ & $17.1 \pm 0.6$ & $21.1 \pm 0.8$ & $1.4 \pm 0.2$ & $2.6 \pm 0.3$ \\
$\mathrm{~N} 4-40 \%$ & $2.4 \pm 0.6$ & $36.4 \pm 7.5$ & $7.3 \pm 1.0$ & $18.6 \pm 2.3$ & $25.5 \pm 5.8$ & $1.7 \pm 0.5$ & $3.2 \pm 0.8$ \\
$\mathrm{~N} 4-50 \%$ & $2.0 \pm 0.3$ & $27.0 \pm 2.5$ & $7.1 \pm 0.3$ & $19.4 \pm 0.4$ & $30.8 \pm 3.0$ & $2.0 \pm 0.3$ & $3.5 \pm 0.3$ \\
$\mathrm{~N} 4-60 \%$ & $3.2 \pm 0.2$ & $32.0 \pm 1.3$ & $4.6 \pm 0.4$ & $10.9 \pm 0.7$ & $82.4 \pm 2.1$ & $7.0 \pm 0.7$ & $12.0 \pm 1.1$ \\
\hline \multicolumn{7}{c}{ M: $80 \%$ methanol, E: $70 \%$ ethanol, \% next to N1 or $\mathrm{N} 4$ is the percentage of added water to NADES (w/w). }
\end{tabular}

In this study, the extraction of phenolic compounds from S. baicalensis bark with citric acid-based NADES was explored with the expectation of providing further evidence for the development and establishment of NADES as universal green solvents for natural products. Proving that NADES are a viable alternative to conventional organic solvents (e.g., aqueous ethanol and methanol) for the extraction of flavonoids was a step in this direction. The highest increase in total flavonoids yield was obtained with N1: citric acid- $\beta$-alanine (1:1, mole/mole) with $50 \%$ water $(w / w)$. N4: citric acid-proline (1:1, mole/mole) with $40 \%$ water. It is noteworthy that both $\mathrm{N} 1$ and N4 contained an amino acid as one of its ingredients, which might be advantageous as food ingredients. Among the tested flavonoids, the extractability of wogonin with $\mathrm{N} 4$ with $60 \%$ water $(w / w)$ was particularly notable, with a 6.32 -fold increase compared to aqueous methanol.

We report the use of a simple method that allows the simultaneous analysis of multiple extracts obtained with different NADES using HPTLC and HPLC. The viscosity of the NADES is still a major obstacle in the recovery of metabolites from the extracts and efforts to provide a technique or process to improve this should be encouraged. The extended range of hydrophilicity/lipophilicity of the extracted compounds achieved in this case with the NADES (with contents between 20 and up to $60 \%$ of water) is also very promising. 


\section{Materials and Methods}

\subsection{Chemicals and Materials}

All of the chemicals for the preparation of NADES, including $\beta$-alanine, citric acid, glucose, xylitol and proline, were analytical grade and purchased from Sigma Aldrich (St. Louis, MO, USA). Analytical standards of scutellarein, scutellarin, baicalein, baicalin, wogonin, wogonoside, oroxylin A and oroxyloside (> 95\% purity) were purchased from Biopurify (Chengdu, China). HPLC grade acetonitrile, water and formic acid were purchased from Honeywell Riedel-de Haën (Seelze, Germany). Dried roots of Scutellaria baicalensis Geroge were collected in Hubei (Wuhan, China) (voucher Specimen No. NPL-R-KDSB-2051210, NPL), purchased from Kyung-Dong market (Seoul, Republic of Korea), and identified by Dr. Y. H. Choi.

\subsection{Preparation of NADES}

Citric acid-based natural deep eutectic solvents employed in this study were made by mixing the ingredients of each NADES at a fixed molar ratio (1:1). These were $\beta$-alanine-citric acid (N1), glucose-citric acid (N2), xylitol-citric acid (N3) and proline-citric acid (N4). The ingredients of NADES were dissolved with a minimum amount of water, followed by heating while stirring at $50{ }^{\circ} \mathrm{C}$. The obtained liquids were dried using a vacuum rotary evaporator at $50{ }^{\circ} \mathrm{C}$ for $4-6 \mathrm{~h}$ (Büchi, Hendrik-Ido-Ambacht, The Netherlands) and residual water was removed by $24 \mathrm{~h}$ of freeze-drying. For the first screening experiment, for the selection of the optimum NADES, 10\% (w/w) of water was added to each NADES, followed by stirring at $40^{\circ} \mathrm{C}$ until a homogenous liquid was formed $[9,26]$.

For the optimization of water content in NADES, N1 ( $\beta$-alanine-citric acid: $1: 1)$ and N4 (proline-citric acid: 1:1) were selected and $20 \%, 30 \%, 40 \%, 50 \%$ and $60 \%(w / w)$ of water was added to each of them, followed by stirring and heating at $50{ }^{\circ} \mathrm{C}$.

\subsection{Extraction of Plant Material}

Mixing of plant material and NADES was performed in a $2 \mathrm{~mL}$-microtube with $100 \mathrm{mg}$ dry weight (DW) of powdered plant material and $2 \mathrm{~mL}$ of NADES. For the control experiments, $70 \%(v / v)$ ethanol or $80 \%(v / v)$ methanol was used instead of the NADES. The mixture was vortexed for 1 min, placed into a water bath (at $40^{\circ} \mathrm{C}$ for $1 \mathrm{~h}$ ), ultrasonicated for $30 \mathrm{~min}$ at room temperature, and centrifuged at 13,000 rpm for $20 \mathrm{~min}$. From the extracts, $1.0 \mathrm{~mL}$ of supernatant was removed and mixed with $1.0 \mathrm{~mL}$ of water and homogenized/vortexed for $1 \mathrm{~min}$. This mixture was submitted to a sample preparation/clean-up process using solid phase extraction (SPE). An aliquot of $1.0 \mathrm{~mL}$ of the control extracts obtained with aqueous ethanol and methanol was dried in a vacuum concentrator and redissolved in $2 \mathrm{~mL}$ of water before applying it on the SPE column. All extracts were prepared by triplicate.

\subsection{Recovery of Compounds from NADES with Solid Phase Extraction (Sample Clean Up)}

Solid phase extraction (SPE) was carried out in $5 \mathrm{~mL}$ Oasis Hydrophilic-Lipophilic-Balance (HLB) cartridges (Waters Corporation, Milford, MA, USA). The HLB cartridges were placed in a vacuum and equilibrated with $5.0 \mathrm{~mL}$ methanol followed by $5.0 \mathrm{~mL}$ of water. After loading the $2 \mathrm{~mL}$ of the sample, the cartridges were flushed with $19 \mathrm{~mL}$ of water and then eluted with $6.0 \mathrm{~mL}$ of methanol. The methanol fractions were dried in a rotary evaporator and redissolved with $2.0 \mathrm{~mL}$ of methanol. Of these fractions, $1.0 \mathrm{~mL}$ was used for the HPTLC analysis and the other $1 \mathrm{~mL}$ for HPLC analysis.

\subsection{HPTLC Analysis}

A CAMAG high performance thin layer chromatography (HPTLC) system equipped with an automatic TLC sampler (version 4), a derivatizer device (version 1.0 AT), a TLC plate heater (version III) and a TLC visualizer (CAMAG, Muttenz, Switzerland) was used for analysis. The separation was 
carried out on $20 \times 10 \mathrm{~cm}$ F254 silica gel 60HPTLC plates (Merck, Darmstadt, Germany). Five $\mu \mathrm{L}$ of the solution were spotted in $6 \mathrm{~mm}$ bands with a distance of $10 \mathrm{~mm}$ from the bottom, $20 \mathrm{~mm}$ from the left and right edge, the distance between bands was $8.8 \mathrm{~mm}$, allowing 18 samples per plate. Two different mobile phases were used for the analysis: ethyl acetate, formic acid, acetic acid and water (100:11:11:27, $v / v / v / v)$ and ethyl acetate, methanol, formic acid and water (20:2.7:0.5:2, $v / v / v / v)$ with a chamber saturation time of $20 \mathrm{~min}$. The developed plates were dried with an air-stream at room temperature and scanned with the CAMAG TLC visualizer at 254, $366 \mathrm{~nm}$ and white light. The plates were also derivatized with $2 \mathrm{~mL}$ of a solution of $1 \mathrm{~g}$ of 2-aminoethyl diphenylborinate in $100 \mathrm{~mL}$ of methanol (NP reagent) and after $5 \mathrm{~min}$ of reaction viewed at $366 \mathrm{~nm}$ with a CAMAG TLC visualizer.

\subsection{HPLC Analysis}

An Agilent Technologies Series 1200 chromatographic system (Santa Clara, CA, USA) equipped with a photodiode array detector (DAD) was used for HPLC analysis. Analysis was performed on a Luna C18 (2) $(100 \mathrm{~mm} \times 4.6 \mathrm{~mm}, 5 \mu \mathrm{m})$ (Phenomenex, Utrecht, The Netherlands) column and samples were eluted with a gradient of $0.1 \%$ formic acid in water (solvent $A$ ) and $0.1 \%$ formic acid in acetonitrile (solvent B) as follows: 15\% B (0-13 min), 15-75\% B (13-14 min), 75-15\% B (14-14.10 min), $15 \%(14.10-15.10 \mathrm{~min})$ at a flow rate of $1 \mathrm{~mL} / \mathrm{min}$. The injection volume for reference compounds and samples was $10 \mu \mathrm{L}$. The chromatogram was recorded at $280 \mathrm{~nm}$ and $340 \mathrm{~nm}$ for quantitation. All HPLC analyses were conducted in triplicate.

For water content optimization analyses, a different column, Phenomenex Luna C18(2) $(150 \mathrm{~mm}$ $\times 4.6 \mathrm{~mm}, 5 \mu \mathrm{m}$ ), was used with the same solvents and gradient program as mentioned above.

\subsection{Data Processing of HPTLC and HPLC Data and Multivariate Data Analysis}

The HPTLC chromatogram scans were processed using the online rTLC software [27]. The generated numerical data of the gray-scale was used in the Rf range between 0.03 and 0.958 . The HPTLC data of chromatograms obtained with both mobile phases were analyzed with the SIMCA-P software (version 15, Umetrics, Umeå, Sweden) for principal component analysis (PCA) using Pareto or UV scaling method.

Author Contributions: Y.H.C. conceived and designed the experiments; W.W.O. and P.B. conducted the experiments under supervision of N.R.M. and E.G.W.; E.G.W. optimized the HPLC method used in the study and gave technical suggestions; W.W.O. analyzed the data and wrote the draft manuscript; Y.H.C. and R.V. reviewed the draft manuscript. All authors have read and agreed to the published version of the manuscript.

Funding: This research received no external funding.

Acknowledgments: The authors thank R. Verpoorte for his interesting thoughts and comments on the manuscript during coffee break discussions.

Conflicts of Interest: The authors declare no conflict of interest.

\section{References}

1. Rogers, R.D.; Seddon, K.R. Ionic Liquids - Solvents of the future? Science 2003, 302, 792-793. [CrossRef] [PubMed]

2. Murtada, K. Trends in nanomaterial-based solid-phase microextraction with a focus on environmental applications-A review. Trends Environ. Anal. Chem. 2019, in press. [CrossRef]

3. Socas-Rodríguez, B.; González-Sálamo, J.; Hernández-Borges, J.; Rodríguez-Delgado, Á. Recent applications of nanomaterials in food safety. TrAC Trends Anal. Chem. 2017, 96, 172-200. [CrossRef]

4. Ahmadi, M.; Elmongy, H.; Madrakian, T.; Abdel-Rehim, M. Nanomaterials as sorbents for ample preparation in bioanalysis: A review. Anal. Chim. Acta 2017, 958, 1-21. [CrossRef] [PubMed]

5. Huang, J.; Guo, X.; Fan, L.; Zhou, X.; Wu, S. Ionic deep eutectic solvents for the extraction and separation of natural products. J. Chromatogra. A 2019, 1598, 1-19. [CrossRef] [PubMed] 
6. Abbott, A.P.; Capper, G.; Davies, D.L.; Munro, H.L.; Rasheed, R.K.; Tambyrajah, V. Preparation of novel, moisture-stable, lewis-acidic ionic liquids containing quaternary ammonium salts with functional side chains. Chem. Commun. 2001, 19, 2010-2011. [CrossRef]

7. Smith, E.L.; Abbott, A.P.; Ryder, K.S. Deep eutectic solvents (DESs) and their applications. Chem. Rev. 2014, 21, 11060-11082. [CrossRef]

8. Zhang, Q.; De Oliveira, V.K.; Royer, S.; Jérome, F. Deep eutectic solvents: Synthesis, properties and applications. Chem. Soc. Rev. 2012, 41, 7108-7146. [CrossRef]

9. Choi, Y.H.; van Spronsen, J.; Dai, Y.; Verberne, M.; Hollmann, F.; Arends, I.W.C.E.; Witkamp, G.-J.; Verpoorte, R. Are natural deep eutectic solvents the missing link in understanding cellular metabolism and Physiology? Plant Physiol. 2011, 156, 1701-1705. [CrossRef]

10. Jeong, K.M.; Zhao, J.; Jin, Y.; Heo, S.R.; Han, S.Y.; Yoo, D.E.; Lee, J. Highly efficient extraction of anthocyanins from grape skin using deep eutectic solvents as green and tunable media. Arch. Pharm. Res. 2015, 38, 2143-2152. [CrossRef]

11. Du, F.Y.; Xiao, X.H.; Li, G.K. Application of ionic liquids in the microwave-assisted extraction of trans-resveratrol from Rhizma Polygoni Cuspidati. J. Chromatogr. A 2007, 1140, 56-62. [CrossRef] [PubMed]

12. Dai, Y.; Witkamp, G.J.; Verpoort, R.; Choi, Y.H. Natural deep eutectic solvents as a new extraction media for phenolic metabolites in Carthamus tincorius L. Anal. Chem. 2013, 85, 6272-6278. [CrossRef] [PubMed]

13. Panic, M.; Gunjevic, V.; Cravotto, G.; Redovnikovic, I.R. Enabling technologies for the extraction of grape pomace anthocyanins using natural deep eutectic solvents in up-to-half-litre batches extraction of grape-pomace anthocyanis using NADES. Food Chem. 2019, 300, 125185. [CrossRef] [PubMed]

14. Bakirtzi, C.; Triantafyllidou, K.; Makris, D.P. Novel lactic acid-based natural deep eutectic solvents: Efficiency in the ultrasound-assisted extraction of antioxidant polyphenols from common native Greek medicinal plants. J. Appl. Res. Med. Aromat. Plants 2016, 3, 120-127. [CrossRef]

15. Dai, Y.; Verpoorte, R.; Choi, Y.H. Natural deep eutectic solvents providing enhanced stability of natural colorants from safflower (Carthamus tinctorius). Food Chem. 2014, 159, 116-121. [CrossRef]

16. Dai, Y.; Witkamp, G.J.; Verpoorte, R.; Choi, Y.H. Tailoring properties of natural deep eutectic solvents with water to facilitate their applications. Food Chem. 2015, 187, 14-19. [CrossRef]

17. Vanda, H.; Dai, Y.; Wilson, E.G.; Verpoorte, R.; Choi, Y.H. Green solvents from ionic liquids and deep eutectic solvents to natural deep eutectic solvents. CR. Chimie 2018, 21, 628-638. [CrossRef]

18. Chen, Z.; Nihei, K.; Tanaka, H.; Uda, Y.; Kabuyama, Y. Identification of a Nitric Oxide Generation-Stimulative Principle in Scutellariae radix. Biosci. Biotechnol. Biochem. 2013, 77, 657-659. [CrossRef]

19. Yoon, S.-B.; Lee, Y.-J.; Park, S.K.; Kim, H.-C.; Bae, H.; Kim, H.M.; Ko, S.-G.; Choi, H.Y.; Oh, M.S.; Park, W. Anti-inflammatory effects of Scutellaria baicalensis water extract on LPS-activated RAW 264.7 macrophages. J. Ethnopharmacol. 2009, 125, 286-290. [CrossRef]

20. Gao, Z.; Yang, X.; Huang, K.; Xu, H. Free-radical scavenging and mechanism study of flavonoids extracted from the radix of Scutellaria baicalensis. Georgi. Appl. Magn. Reson. 2000, 19, 35-44. [CrossRef]

21. Liu, X.; Ahlgren, S.; Korthout, H.A.A.J.; Salomé-Abarca, L.F.; Bayona, L.M.; Verpoorte, R.; Choi, Y.H. Broad range chemical profiling of natural deep eutectic solvent extracts using a high performance thin layer chromatography-based method. J. Chromatogr. A 2018, 1532, 198-207. [CrossRef] [PubMed]

22. Ge, Y.; Sun, M.; Salomé-Abarca, L.F.; Wang, M.; Choi, Y.H. Investigation of species and environmental effects on Rhubarb roots metabolome using ${ }^{1} \mathrm{H}$ NMR combined with high performance thin layer chromatography. Metabolomics 2018, 14, 137. [CrossRef] [PubMed]

23. Salomé-Abarca, L.F.; Van der Pas, J.; Kim, H.K.; Van Uffelen, G.A.; Klinkhamer, P.G.L.; Choi, Y.H. Phytochemistry 2018, 155, 37-44.

24. González, C.G.; Mustafa, N.R.; Wilson, E.G.; Verpoorte, R.; Choi, Y.H. Application of natural deep eutectic solvents for the "green" extraction of vanillin from vanilla pods. Flavour Fragr. J. 2018, 33, 91-96. [CrossRef]

25. Liu, G.; Rajesh, N.; Wang, X.; Zhang, M.; Wu, Q.; Li, S.; Chen, B.; Yao, S. Identification of flavonoids in the stems and leaves of Scutellaria baicalensis. Georgi. J. Chromatogr. B. 2011, 879, 1023-1028. [CrossRef] [PubMed]

26. Dai, Y.; van Spronsen, J.; Witkamp, G.J.; Verpoorte, R.; Choi, Y.H. Natural deep eutectic solvents as new potential media for green technology. Anal. Chim. Acta 2013, 766, 61-68. [CrossRef] [PubMed]

27. Fichou, D.; Ristivojevic, P.; Morlock, G.E. Proof-of-principle of rTLC, an open-source software developed for image evaluation and multivariate analysis of planar chromatograms. Anal. Chem. 2016, 88, 12494-12501. [CrossRef] 
Sample Availability: Samples of the compounds (baicalein, scutellarein, wogonin, oroxylin A, baicalin, scutellarin, wogonoside and oroxyloside) are available from the authors.

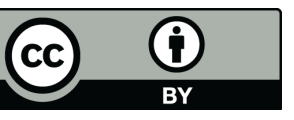

(C) 2020 by the authors. Licensee MDPI, Basel, Switzerland. This article is an open access article distributed under the terms and conditions of the Creative Commons Attribution (CC BY) license (http://creativecommons.org/licenses/by/4.0/). 